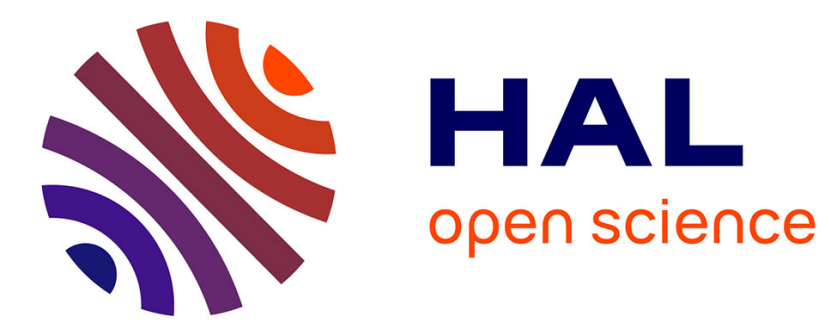

\title{
Estimating the state and the unknown inputs of nonlinear systems using a multiple model approach
}

\author{
Rodolfo Orjuela, Benoît Marx, José Ragot, Didier Maquin
}

\section{To cite this version:}

Rodolfo Orjuela, Benoît Marx, José Ragot, Didier Maquin. Estimating the state and the unknown inputs of nonlinear systems using a multiple model approach. 16th Mediterranean Conference on Control and Automation, MED'08, Jun 2008, Ajaccio, France. pp.1375-1380, 10.1109/MED.2008.4602077 . hal-00278921

\section{HAL Id: hal-00278921 \\ https://hal.science/hal-00278921}

Submitted on 5 May 2014

HAL is a multi-disciplinary open access archive for the deposit and dissemination of scientific research documents, whether they are published or not. The documents may come from teaching and research institutions in France or abroad, or from public or private research centers.
L'archive ouverte pluridisciplinaire HAL, est destinée au dépôt et à la diffusion de documents scientifiques de niveau recherche, publiés ou non, émanant des établissements d'enseignement et de recherche français ou étrangers, des laboratoires publics ou privés. 


\title{
Estimating the state and the unknown inputs of nonlinear systems using a multiple model approach
}

\author{
Rodolfo Orjuela, Benoît Marx, José Ragot and Didier Maquin
}

\begin{abstract}
This paper addresses both state and unknown input estimation problem of nonlinear systems modelled with the help of a particular class of multiple models, known as decoupled multiple model. The simultaneous estimation of the state and the unknown inputs is achieved using a proportionalintegral observer that is well known by its robustness properties. The proposed observer allows the use of submodels with different dimensions and this fact offers potential applications in the multiple model framework. The LMI framework is used in order to provide sufficient conditions for ensuring exponential convergence of the estimation error and robust $\mathscr{H}_{\infty}$ performances with respect to perturbations.
\end{abstract}

\section{INTRODUCTION}

The simultaneous estimation of the state and the unknown inputs (UI) of a system is a key problem in many engineering applications due to the practical and/or economical problems arising when measuring signals of a process. A robust state estimation with respect to UI plays therefore a fundamental role in numerous system control and/or supervision strategies. With regard to this last purpose, an UI can generally be employed in order to modelling an actuator failure and/or an abnormal behaviour of an internal component of the system. Clearly, the state and the UI estimations can be employed for providing fault symptoms of the systems in order to make the system more reliable and safe.

Classically, a state estimation of a system subject to UI can be obtained by means of the so called unknown input observer (UIO). The goal of the UIO is to provide state reconstruction of the system with some robustness with respect to possible UI. Design of UIO has been extensively investigated in the literature and design procedures for reduced-order UIO [1] and full-order UIO [2] have been proposed (see [3] for a comprehensive study of UI observer problem). Recently, in [4] a projection operator approach is used to design full and reducer-order UIO. Comparison and relation between the proposed observer and other classic UIO architectures are also proposed. Note however that the UI estimation is not considered in these works.

In [5] is shown that the UI reconstruction is very close to the system inversion. Hence, derivatives of the measurements must be used in the UI reconstruction problem. Consequently, UI estimation sensibility with respect to measurements noise is often unavoidable. In [6] the asymptotic

This work is partially supported by the Conseil Régional de Lorraine (France) and by the TASSILI n.07 program under MDU grant 714 .

The authors are with Centre de Recherche en Automatique de Nancy (CRAN), Nancy-Université, CNRS, 2 avenue de la Forêt de Haye F-54516, Vandœuvre-lès-Nancy \{rodolfo.orjuela, benoit.marx, jose.ragot, didier.maquin\} densem.inpl-nancy.fr system state and UI estimation with any desired accuracy is proposed. In this approach the use of time derivative of the measurements is avoided.

The Proportional-Integral Observer (PIO) constitutes an other attractive way to solve the UI problem without involving the time derivative of the measurements. The PIO is well known by its robustness properties [7], [8] and it has been successfully employed in order to cope with the state and the UI estimations [9]-[12]. It has been established that, in the context of system supervision, the PIO can be used as robust residual generator [11], [13].

However, most of these works assume that the system has a linear behaviour whereas in practice many processes have generally a nonlinear dynamic behaviour. Hence the use of a single linear model for modelling the dynamic behaviour of a system in the whole operating space can be unsuited because the linearity assumption of the system is only valid in the neighbourhood of an operating point. On the other hand, the observer design problem for generic nonlinear models is delicate and actually this problem is not solved in a general way.

Multiple models are an effective tool to accurately represent nonlinear dynamic behaviours using a model structure potentially usable for extending, in an elegant way, some results obtained in the linear control theory to nonlinear systems. In this modelling strategy the complexity of the system is reduced by a decomposition of the operating range of the system in a finite number of operating zones [14]. Each operating zone is then characterised by a submodel, often a linear model, and the global model is obtained by interpolating the submodels via a weighting function.

A multiple model can therefore be viewed as an association of a set of submodels blended by an interpolation mechanism. As pointed in [15], different multiple models can be obtained using different aggregation structures of the submodels. Basically, two main structures can be distinguished. In the first one, the submodels share the same state vector (Takagi-Sugeno multiple model); in the second one, the submodels are decoupled and their state vectors are different (decoupled multiple model). Of course, the resulting multiple models obtained by the association of the same set of submodels do not give an equivalent dynamic behaviour.

The Takagi-Sugeno model has been largely considered for analysis, modelling, control and state estimation of nonlinear systems (see among others [14], [16]-[18] and references therein). Structural similarities between this multiple model and others models, for example linear parameter varying models (LPV), have been established in the literature [19]. 
Indeed, in this multiple model the submodel association is performed in the dynamic equation of the model via a common state vector. Consequently, the dimension of the submodels must be identical. Hence, the use of this multiple model in some black box modelling problems can lead to a redundant multiple model because the submodel dimensions must be identical in each operating zone even if a low dimension submodel can be used in a particular zone.

The second multiple model structure can be expected to be more flexible in the modelling stage because the aggregation of submodels is performed in such a way that the dimension of the submodels may be different. Therefore the dimension of each submodel can be well adapted to each operating zone (details are given in section 2). Note that in contrast to the Takagi-Sugeno form, less attention has been paid in the literature to the decoupled multiple model. However, few works in modelling [20], [21], control [22]-[24] and state estimation [25], [26] of nonlinear systems have made a successful implementation of this structure and shown its relevance.

The main contribution of this paper is the extension of the proportional-integral observer design procedure used in the linear theory to the nonlinear systems modelled by a decoupled multiple model. Exponential convergence is obtained in the disturbance free case and in the presence of UI, $\mathscr{H}_{\infty}$ performance is assured.

The paper is organized as follows. The decoupled multiple model is presented in section 2. Preliminaries and problem statement are presented in section 3 . In section 4 , the robust $\mathscr{H}_{\infty}$ observer design is investigated and the gains of the observer are obtained by LMI optimization. Finally, in section 5, a simulation example illustrates the state and UI estimations of a decoupled multiple model.

\section{ON THE DECOUPLED MULTIPLE MODEL}

The multiple model exploits the idea that complex dynamic behaviours can be accurately represented with the help of an interpolation of simple submodels. In this modelling framework the aggregation of these submodels can be performed using several ways. The decoupled multiple model is among them [15]. In this paper, it is slightly modified using a state space representation as follows:

$$
\begin{aligned}
\dot{x}_{i}(t) & =A_{i} x_{i}(t)+B_{i} u(t)+D_{i} \eta(t)+V_{i} w(t), \\
y_{i}(t) & =C_{i} x_{i}(t), \\
y(t) & =\sum_{i=1}^{L} \mu_{i}(\xi(t)) y_{i}(t)+E \eta(t)+W w(t),
\end{aligned}
$$

where $x_{i} \in \mathbb{R}^{n_{i}}$ and $y_{i} \in \mathbb{R}^{p}$ are respectively the state vector and the output of the $\mathrm{i}^{\text {th }}$ submodel; $u \in \mathbb{R}^{m}$ is the known input, $\eta \in \mathbb{R}^{q}$ the unknown input, $y \in \mathbb{R}^{p}$ the measured output and $w \in \mathbb{R}^{r}$ the perturbation. The matrices $A_{i} \in \mathbb{R}^{n_{i} \times n_{i}}$, $B_{i} \in \mathbb{R}^{n_{i} \times m}, D_{i} \in \mathbb{R}^{n_{i} \times q}, V_{i} \in \mathbb{R}^{n_{i} \times r}, C_{i} \in \mathbb{R}^{p \times n_{i}}, E \in \mathbb{R}^{p \times q}$ and $W \in \mathbb{R}^{p \times r}$ are known and appropriately dimensioned.

The so called decision variable signal $\xi(t)$ is assumed to be known and real-time available (e.g. the inputs, the outputs and exogenous signals). The contribution of the submodels are quantified by the weighting functions $\mu_{i}(\xi(t))$ that satisfy the following convex sum constraints:

$$
\sum_{i=1}^{L} \mu_{i}(\xi(t))=1 \text { and } 0 \leq \mu_{i}(\xi(t)) \leq 1, \forall i=1 \ldots L, \forall t .
$$

It should be mentioned that the weighting functions may take intermediary values over the range 0 to 1 . This is due to possible overlapping of operating zones. Therefore the multiple model has a true nonlinear dynamic behaviour instead of a piecewise linear behaviour.

As pointed in the introduction, in this multiple model no blend between the parameters of the submodels is performed. Consequently, the dimension (i.e. the number of states) of the submodels can be different and therefore this multiple model form is suitable for a black box modelling of complex systems with variable structure and/or variable complexity in each operating zone. The model parameters can be obtained from a set of measured input and output data using appropriate identification methods proposed for instance in [20], [21], [27].

Besides, in this multiple model, the outputs $y_{i}(t)$ of the submodels are "virtual outputs", i.e. no physical interpretation is available. These outputs must be considered as artificial modelling signals only used in the goal to provide a representation of the real system behaviour. Consequently the outputs $y_{i}(t)$ cannot be viewed as accessible signals and therefore they cannot be employed for driving an observer.

Finally, let us remark that in some particular situations, a discontinuity in the multiple model output appears when a submodel that is away of the current operating zone is suddenly taken into consideration for building the multiple model output. Of course, this phenomenon does not appear in a systematic way and it depends on the dynamics of the submodels and/or on the choice of the decision variable. When the input of the system is employed as decision variable, this undesirable phenomenon can effectively be overcame by using a filtered value of the decision variable instead of its direct value. Usually, a low-pass filter with unit gain is employed. The parameters of this filter are obtained using a priori knowledge of the system [23] or by considering them as unknown parameters in the identification stage [27].

Notations: the following notations will be used all along this paper. $P>0(P<0)$ denotes a positive (negative) definite matrix $P ; X^{T}$ denotes the transpose of matrix $X, \mathrm{I}$ is the identity matrix of appropriate dimension and $\operatorname{diag}\left\{A_{1}, \ldots, A_{n}\right\}$ stands for a block-diagonal matrix with the matrices $A_{i}$ on the main diagonal. The $L_{2}$-norm of a signal, quantifying its energy is denoted and defined by $\|e(t)\|_{2}^{2}=\int_{0}^{\infty} e^{T}(t) e(t) d t$. Finally, we shall simply write $\mu_{i}(\xi(t))=\mu_{i}(t)$.

\section{PRELIMINARIES AND PROBLEM FORMULATION}

Let us consider the decoupled multiple model (1) in the following compact form:

$$
\begin{aligned}
\dot{x}(t) & =\tilde{A} x(t)+\tilde{B} u(t)+\tilde{D} \eta(t)+\tilde{V} w(t) \\
y(t) & =\tilde{C}(t) x(t)+E \eta(t)+W w(t)
\end{aligned}
$$


where:

$$
\begin{aligned}
\tilde{A} & =\operatorname{diag}\left\{A_{1} \cdots A_{i} \cdots A_{L}\right\} \\
\tilde{B} & =\left[B_{1}{ }^{T} \cdots B_{i}^{T} \cdots B_{L}^{T}\right]^{T} \\
\tilde{D} & =\left[D_{1}^{T} \cdots D_{i}^{T} \cdots D_{L}^{T}\right]^{T} \\
\tilde{V} & =\left[V_{1}^{T} \cdots V_{i}^{T} \cdots V_{L}^{T}\right]^{T} \\
\tilde{C}(t) & =\left[\mu_{1}(t) C_{1} \cdots \mu_{i}(t) C_{i} \cdots \mu_{L}(t) C_{L}\right] \\
x(t) & =\left[x_{1}^{T}(t) \cdots x_{i}^{T}(t) \cdots x_{L}^{T}(t)\right]^{T} \in \mathbb{R}^{n}, n=\sum_{i=1}^{L} n_{i} .
\end{aligned}
$$

The reader may have noticed that the matrix $\tilde{C}(t)$ can be rewritten as follows:

$$
\tilde{C}(t)=\sum_{i=1}^{L} \mu_{i}(t) \tilde{C}_{i}
$$

where $\tilde{C}_{i}$ is a constant block matrix given by:

$$
\tilde{C}_{i}=\left[\begin{array}{ll}
0 \cdots & C_{i} \cdots 0
\end{array}\right] .
$$

Our objective is to provide a simultaneous estimation of the state and the UI of the system (3). To this end, the following PIO is employed:

$$
\begin{aligned}
\dot{\hat{x}}(t) & =\tilde{A} \hat{x}(t)+\tilde{B} u(t)+\tilde{D} \hat{\eta}(t)+\tilde{K}(y(t)-\hat{y}(t)), \\
\dot{\hat{\eta}}(t) & =\tilde{K}_{1}(y(t)-\hat{y}(t)) \\
\hat{y}(t) & =\tilde{C}(t) \hat{x}(t)+E \hat{\eta}(t) .
\end{aligned}
$$

The use of an integral action given in the second equation of (12) is at the origin of the designation Proportional-Integral Observer. The use of this integral action allows a reconstruction of the UI under the following two assumptions:

Assumption 1: The unknown input signal $\eta(t)$ is supposed to be a constant signal.

Assumption 2: The perturbation is bounded energy signal, i.e. $\|w(t)\|_{2}^{2}<\infty$.

Now, let us define the state estimation error by:

$$
e(t)=x(t)-\hat{x}(t),
$$

and its time-derivative by:

$$
\begin{aligned}
\dot{e}(t) & =\sum_{i=1}^{L} \mu_{i}(t)\left(\tilde{A}-\tilde{K} \tilde{C}_{i}\right) e(t)+(\tilde{D}-\tilde{K} E) \varepsilon(t) \\
& +(\tilde{V}-\tilde{K} W) w(t) .
\end{aligned}
$$

where $\varepsilon(t)$ is the UI estimation error given by:

$$
\varepsilon(t)=\eta(t)-\hat{\eta}(t),
$$

and its time-derivative is given by:

$$
\dot{\varepsilon}(t)=\dot{\eta}(t)-\tilde{K}_{1} \tilde{C}(t) e(t)-\tilde{K}_{1} E \varepsilon(t)-\tilde{K}_{1} W w(t) .
$$

Note that the first term of the right-hand side of the above equation vanishes by considering the assumption 1:

$$
\dot{\varepsilon}(t)=-\tilde{K}_{1} \tilde{C}(t) e(t)-\tilde{K}_{1} E \varepsilon(t)-\tilde{K}_{1} W w(t) .
$$

Finally, by introducing the following augmented vector:

$$
\Sigma(t)=\left[\begin{array}{ll}
e^{T}(t) & \varepsilon^{T}(t)
\end{array}\right]^{T} \in \mathbb{R}^{n+q},
$$

the equations (14) and (17) can be gathered as follows:

$$
\dot{\Sigma}(t)=\tilde{A}_{a}(t) \Sigma(t)+\left(V_{a}-K_{a} W\right) w(t),
$$

where

$$
\begin{aligned}
\tilde{A}_{a}(t) & =\sum_{i=1}^{L} \mu_{i}(t) \Phi_{i} \\
\Phi_{i} & =A_{a}-K_{a} \mathscr{C}_{i}
\end{aligned}
$$

and

$$
A_{a}=\left[\begin{array}{cc}
\tilde{A} & \tilde{D} \\
0 & 0
\end{array}\right], K_{a}=\left[\begin{array}{c}
\tilde{K} \\
\tilde{K}_{1}
\end{array}\right], \mathscr{C}_{i}=\left[\begin{array}{c}
\tilde{C}_{i}^{T} \\
E^{T}
\end{array}\right]^{T}, V_{a}=\left[\begin{array}{c}
\tilde{V} \\
0
\end{array}\right] .
$$

The robust observer design problem can thus be formulated as finding the matrix gain $K_{a} \in \mathbb{R}^{(n+p) \times p}$ such that the influence of $w(t)$ on $\Sigma(t)$ is attenuated. Now let us consider the following objective signal:

$$
z(t)=H \Sigma(t),
$$

where $H$ is a prescribed constant matrix and the following $\mathscr{H}_{\infty}$ performance constraints:

$$
\begin{aligned}
\lim _{t \rightarrow \infty} \Sigma(t)=0 & \text { for } \quad w(t)=0, \\
\|z(t)\|_{2}^{2} \leq \gamma^{2}\|w(t)\|_{2}^{2} & \text { for } \quad w(t) \neq 0 \text { and } z(0)=0,
\end{aligned}
$$

where $\gamma$ is the $L_{2}$ gain from $w(t)$ to $z(t)$ to be minimised. The matrix $H$ in (23) is used in order to take into consideration totally or partially the components of the estimation error $\Sigma(t)$ given by (18). Notice that the condition (24a) will be investigated by imposing the exponential convergence of the estimation error. The exponential convergence of the estimation error is a way to ensure a convergence velocity of the estimation error via a decay rate and to improve dynamic performances of the observer.

\section{UNKNOWN INPUT OBSERVER DESIGN}

This section deals with the $\mathscr{H}_{\infty}$ estimation problem, based on the decoupled multiple model (3), using the PIO (12).

It should be noted that, in the multiple model framework, an unstable multiple model can be obtained via the interpolation of a set of stable submodels and a stable multiple model can be obtained via an interpolation of a set of unstable submodels. Hence, independent observer designs for each submodel cannot guarantee the global convergence of the estimation error (19). Indeed, the blending between the submodel outputs must be taken into consideration in the observer design.

In this section, conditions for ensuring the estimation error convergence, under constraints (24), are established in LMI framework [28] using a quadratic Lyapunov function. Our main result is summarized in the following theorem:

Theorem 1: The PI observer (12) for the decoupled multiple model (3), under constraints (24), is obtained if there exists a symmetric, positive definite matrix $P$ and a matrix $M$ minimizing $\bar{\gamma}>0$ under the following LMIs

$$
\left[\begin{array}{cc}
\Delta_{i}+\Delta_{i}^{T}+H^{T} H & \Gamma \\
\Gamma^{T} & -\bar{\gamma} \mathrm{I}
\end{array}\right]<0, \quad i=1 \ldots L
$$


where

$$
\begin{aligned}
\Delta_{i} & =P\left(A_{a}+\alpha \mathrm{I}\right)-M \mathscr{C}_{i} \\
\Gamma & =P V_{a}-M W
\end{aligned}
$$

for a prescribed $\alpha>0$.

The observer gain is given by $K_{a}=P^{-1} M$ and the $L_{2}$ gain from $w(t)$ to $z(t)$ is given by $\gamma=\sqrt{\bar{\gamma}}$.

Proof: Let us consider the quadratic Lyapunov function:

$$
V(t)=\Sigma^{T}(t) P \Sigma(t), \quad P>0 \quad P=P^{T},
$$

classically, the exponential convergence of the estimation error $\Sigma(t)$ when $w(t)=0$ is investigated by taking into consideration the following condition:

$$
\dot{V}(t)<-2 \alpha V(t),
$$

where $\alpha$ is the so called decay rate. Here, the above condition is replaced by the following condition:

$$
\dot{V}(t)<-2 \alpha V(t)-z^{T}(t) z(t)+\gamma^{2} w^{T}(t) w(t) .
$$

in order to guaranteed robust performances (24). Indeed, it can be noted that the integration of condition (28) yields:

$$
\begin{aligned}
\int_{0}^{\infty}(\dot{V}(t)+2 \alpha V(t)) d t & <-\int_{0}^{\infty} z^{T}(t) z(t) d t \\
& +\gamma^{2} \int_{0}^{\infty} w^{T}(t) w(t) d t
\end{aligned}
$$

and by taking into consideration the positivity of the Lyapunov function, $V(\infty)>0$ and $V(0)=0$, the left-hand side of this inequality is positive and implies that:

$$
\|z(t)\|_{2}^{2}<\gamma^{2}\|w(t)\|_{2}^{2},
$$

hence the attenuation level between the perturbation $w(t)$ and the objective signal $z(t)$, given by (24b), is ensured if the condition (28) is fulfilled.

Consequently, conditions verifying (28) must be established in order to satisfy robust performances (24) and exponential convergence of the estimation error.

The time-derivative of (26) along the trajectories of (18) is given by:

$$
\dot{V}(t)=\dot{\Sigma}^{T}(t) P \Sigma(t)+\Sigma^{T}(t) P \dot{\Sigma}(t),
$$

that becomes by using (19):

$$
\begin{aligned}
\dot{V}(t) & =\Sigma^{T}(t)\left(\tilde{A}_{a}^{T}(t) P+P \tilde{A}_{a}(t)\right) \Sigma(t) \\
& +w^{T}(t)\left(V_{a}-K_{a} W\right)^{T} P \Sigma(t) \\
& +\Sigma^{T}(t) P\left(V_{a}-K_{a} W\right) w(t) .
\end{aligned}
$$

The above equation can be rewritten in the following compact form as:

$$
\dot{V}(t)=\psi(t)^{T} \Omega(t) \psi(t)
$$

$$
\begin{aligned}
& \text { where } \\
& \Omega(t)=\left[\begin{array}{cc}
\tilde{A}_{a}^{T}(t) P+P \tilde{A}_{a}(t) & P\left(V_{a}-K_{a} W\right) \\
\left(V_{a}-K_{a} W\right)^{T} P & 0
\end{array}\right], \\
& \psi(t)=\left[\begin{array}{ll}
\Sigma^{T}(t) & w^{T}(t)
\end{array}\right]^{T} .
\end{aligned}
$$

Now, substituting (26) and (33) into (28) yields:

$$
\psi^{T}(t)\left\{\Omega(t)+\left[\begin{array}{cc}
H^{T} H+2 \alpha P & 0 \\
0 & -\gamma^{2} \mathrm{I}
\end{array}\right]\right\} \psi(t)<0,
$$

which is a quadratic form in $\psi(t)$. Therefore the negativity of the above expression is guaranteed by ensuring the negativity of the expression inside the brackets.

Hence, by using (34) and the definition (20) of $\tilde{A}_{a}(t)$, the negativity of (36) is satisfied by:

$$
\sum_{i=1}^{L} \mu_{i}(t)\left[\begin{array}{cc}
\Phi_{i}^{T} P+P \Phi_{i}+H^{T} H+2 \alpha P & P\left(V_{a}-K_{a} W\right) \\
\left(V_{a}-K_{a} W\right)^{T} P & -\gamma^{2} \mathrm{I}
\end{array}\right]<0,
$$

which is also guaranteed according to the convex sum properties of the weighting functions (2) by:

$$
\left[\begin{array}{cc}
\Phi_{i}^{T} P+P \Phi_{i}+H^{T} H+2 \alpha P & P\left(V_{a}-K_{a} W\right) \\
\left(V_{a}-K_{a} W\right)^{T} P & -\gamma^{2} \mathrm{I}
\end{array}\right]<0,
$$

for $i=1 \ldots L$.

Finally, let us notice that this inequality is not a LMI in $P, K_{a}, \alpha$ and $\gamma$. However, it becomes a LMI by choosing a prescribed $\alpha$ and setting $M=P K_{a}$ and $\bar{\gamma}=\gamma^{2}$. Hence standard convex optimization algorithms can be used to find matrices $P$ and $M$ minimising $\bar{\gamma}$, for a prescribed $\alpha$.

On the other hand, the negativity of condition (38) implies the negativity of the block $(1,1)$, consequently:

$$
P\left(A_{a}+\alpha \mathrm{I}-K_{a} \mathscr{C}_{i}\right)+\left(A_{a}+\alpha \mathrm{I}-K_{a} \mathscr{C}_{i}\right)^{T} P<0,
$$

for $i=1 \ldots L$, which means that exponential convergence of the estimation error is guaranteed in the free perturbation case because the matrix $\tilde{A}_{a}(t)$ in (19) is Hurwitz for any blend between the submodel outputs [26]. Hence, the condition (24a) is satisfied under $w(t)=0$ and this completes the proof.

\section{Simulation example}

Consider the following two submodels of a decoupled multiple model:

$$
\begin{array}{rlrl}
A_{1} & =\left[\begin{array}{ccc}
-0.2 & 0.1 & 0 \\
0.2 & -0.9 & 0.8 \\
0 & -0.8 & -0.7
\end{array}\right], & A_{2}=\left[\begin{array}{cc}
-0.25 & 0 \\
-0.4 & -0.3
\end{array}\right], \\
B_{1}=\left[\begin{array}{lll}
0.5 & 0.4 & 0.3
\end{array}\right]^{T}, & B_{2}=\left[\begin{array}{cc}
-0.5 & 0.7
\end{array}\right]^{T}, \\
C_{1}=\left[\begin{array}{ccc}
0.8 & 0.5 & 0.7 \\
0.4 & -0.7 & -0.2
\end{array}\right], & C_{2}=\left[\begin{array}{cc}
0.9 & 0.6 \\
0.5 & -0.4
\end{array}\right], \\
D_{1}=\left[\begin{array}{cc}
0.1 & 0.3 \\
0.2 & 0.4 \\
0 & -0.2
\end{array}\right], & D_{2}=\left[\begin{array}{cc}
0.1 & 0.3 \\
-0.1 & 0.5
\end{array}\right]^{-1}, \\
V_{1}=\left[\begin{array}{ccc}
0.0 & -0.1 & 0.0 \\
0.2 & -0.3 & 0.1
\end{array}\right]^{T}, & V_{2}=\left[\begin{array}{cc}
0.0 & -0.1 \\
0.2 & 0.0
\end{array}\right]^{T}, \\
E & =\left[\begin{array}{cc}
0.1 & 0.2 \\
0.5 & -0.3
\end{array}\right], & W & =\left[\begin{array}{cc}
0.1 & 0 \\
0 & 0.1
\end{array}\right] .
\end{array}
$$

Here, the objective signal $z(t)$ to be attenuated is the state estimation error of the submodels, thus $H=\left[\begin{array}{ll}\mathrm{I}_{(5 \times 5)} & 0_{(5 \times 2)}\end{array}\right]$ in (23). The disturbance and the UI of the system are respectively given by:

$$
w(t)=\left[\begin{array}{c}
0.4 \sin (40 t) \\
0.35 \sin (60 t)
\end{array}\right] \quad, \quad \eta(t)=\left[\begin{array}{l}
\eta_{1}(t) \\
\eta_{2}(t)
\end{array}\right],
$$


where $\eta_{1}(t)$ is a piecewise constant signal for which the assumption 1 is well checked, and $\eta_{2}(t)$ is voluntarily taken as a ramp between $t=600$ and $t=1000$, so the assumption 1 is not fulfilled in this range (see figures 2 and 3 ).

The weighting functions are obtained from normalised Gaussian functions:

$$
\begin{aligned}
& \mu_{i}(\xi(t))=\omega_{i}(\xi(t)) / \sum_{j=1}^{L} \omega_{j}(\xi(t)), \\
& \omega_{i}(\xi(t))=\exp \left(-\left(\xi(t)-c_{i}\right)^{2} / \sigma^{2}\right),
\end{aligned}
$$

with the standard deviation $\sigma=0.5$ and the centres $c_{1}=0.25$ and $c_{2}=0.75$. Here, the decision variable $\xi(t)$ is the filtered input signal $u(t)$ of the system:

$$
\dot{\xi}(t)=-0.1 \xi(t)+0.1 u(t),
$$

and the input signal $u(t)$ is a piecewise constant signal with variable amplitudes in the range of variation $[0,1]$. Note that the weighting functions are not null or equal to one (see figure 1 (bottom)). Consequently the multiple model output is obtained by taking into consideration the contribution of both submodels all the time.
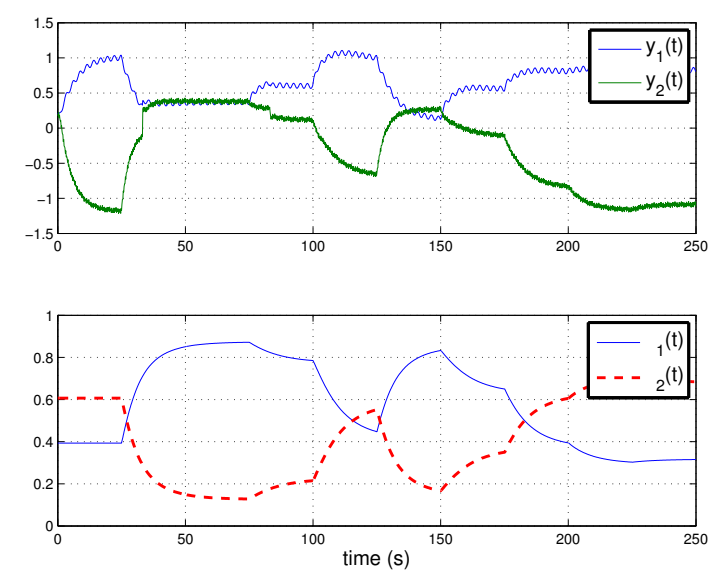

Fig. 1. Multiple model outputs (top) and weighting functions (bottom)

On the other hand, the eigenvalues of the submodels are given by :

$\lambda_{1}=[-0.19-0.80 \pm 0.78 i]$ and $\lambda_{2}=[-0.3-0.25]$,

thus the dynamics of the submodels are different and the dynamic behaviour of the multiple model can be expected to be nonlinear (figure 1 (top)).

A solution to conditions of theorem 1 can be found by using, for example, YALMIP interface [29] coupled to SeDuMi solver [30]. Choosing a decay rate $\alpha=0.1$, conditions of theorem 1 are fulfilled with:

$K_{a}=\left[\begin{array}{lllllll}2.56 & -0.08 & -1.82 & 2.28 & 3.80 & 3.18 & 2.94 \\ 0.95 & -0.64 & -1.29 & 0.81 & 1.64 & 3.34 & 1.07\end{array}\right]^{T}$

with a minimal attenuation level given by $\gamma=1.29$. The state estimation errors obtained by the proposed observer are plotted in figures 2 and 3 and the provided UI estimation in figures 4 and 5 . Let us notice that in the simulation the initial conditions of the multiple model are not null and the initial conditions of the observer are null. It can be seen from these figures that the state estimation error remains globally bounded and close to zero. It is punctually affected when the value of the UI changes abruptly, for example at $t=33$, obviously the assumption 1 is not respected. On the other hand, the proposed observer yields an excellent UI estimation even if the assumption 1 is not truly respected (see figures 4 and 5). Note however that in both case the estimation error has a good transient response.
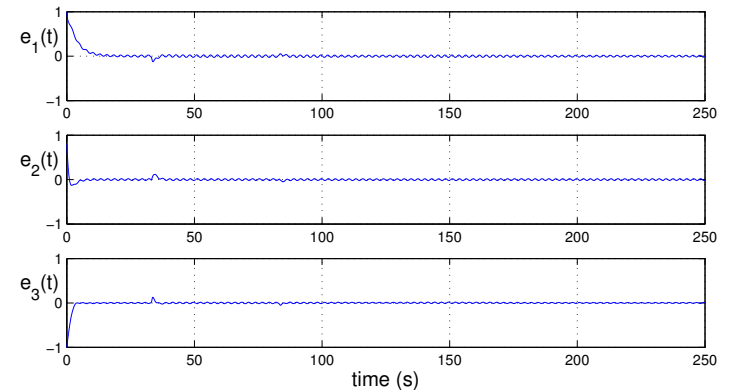

Fig. 2. State estimation errors of submodel 1
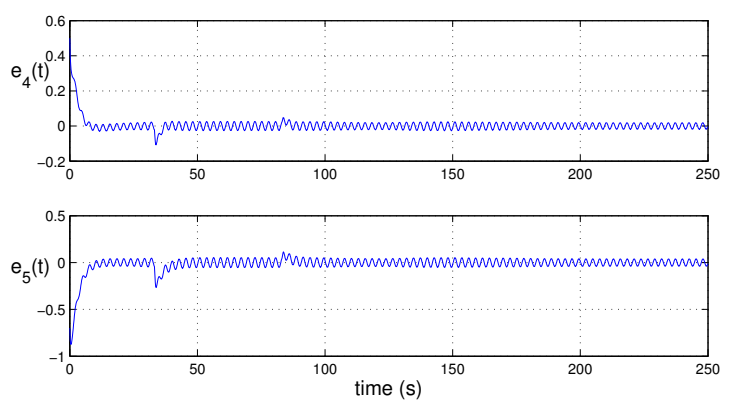

Fig. 3. State estimation errors of submodel 2

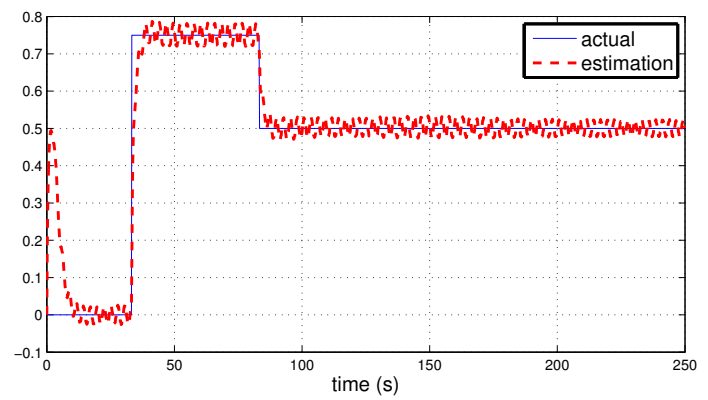

Fig. 4. $\quad \eta_{1}(t)$ and its estimate

As one can see in this example, the assumption 1 is needed for theoretical proofs but our approach remains effective in practical cases where the assumption 1 is not satisfied, of course the UI must be a low frequency signal (constant or slowly varying-time signal). The UI reconstruction can be improved by replacing the use of only one observer by a bank of dedicated observers. Hence each observer is designed using the same procedure but by changing the prescribed 


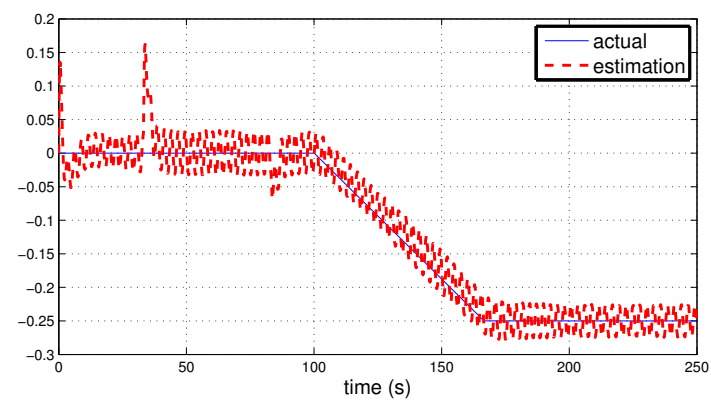

Fig. 5. $\quad \eta_{2}(t)$ and its estimate

matrix $H$ in (23) in order to obtain different attenuation levels. As clearly seen from these pictures, the proposed observer provides good dynamic and robust performances.

\section{CONCLUSION AND Future WORKS}

In the present paper an extension of proportional-integral observer is presented for estimating the state variables and the unknown inputs of nonlinear systems modelled by a decoupled multiple model. Thanks to the structure of the proposed model the number of the states, i.e. the dimension, of each submodel may be different and consequently some flexibility in a black box modelling stage can be is provided. Systematic procedure, based on the LMI framework, has been established in order to design an UI observer which ensures the exponential convergence and robust performances of the estimation error. The effectiveness of the proposed approach is illustrated via a simulation example.

The suggested observer can be used, as an extension of the classic generalized observer scheme, in the detection and the isolation of sensor and actuator failures of complex systems. Improvements to the proposed observer, in order to take into consideration a more general class of unknown inputs, provides promising prospects in the future. In particular, the use of several integral actions by using a Multi-Integral Observer architecture can be an effective way in order to apply the proposed procedure to non constant unknown inputs.

\section{REFERENCES}

[1] M. Hou and P. Muller, "Design of observers for linear systems with unknown inputs." IEEE Transactions on Automatic Control, vol. 37, no. 6 , pp. 871-875, 1992.

[2] M. Darouach, M. Zasadzinski, and S. Xu, "Full-order observers for linear systems with unknown inputs," IEEE Transactions on Automatic Control, vol. 39, no. 3, pp. 606-609, 1994.

[3] M. Witczak, Modelling and estimation strategies for fault diagnosisi of non-linear systems, M. Thoma and M. Morari, Eds. Berlin: SpringerVerlag, 2007.

[4] S. Hui and S. Zak, "Observer design for systems with unknown inputs," International Journal of Applied Mathematics and Computer Science, vol. 15, no. 4, pp. 431-446, 2005.

[5] M. Hou and R. Patton, "Input observability and input reconstruction," Automatica, vol. 34, no. 6, pp. 789-794, 1998.

[6] M. Corless and J. Tu, "State and input estimation for a class of uncertain systems," Automatica, vol. 34, no. 6, pp. 757-764, 1998.

[7] S. Beale and B. Shafai, "Robust control system design with a proportional integral observer," International Journal of Control, vol. 50, no. 1 , pp. 97-111, 1989.
[8] A. Weinmann, Uncertain Models and Robust Control. Vienne: Springer-Verlag Wien New York, 1991.

[9] D. Söffker, "Fault detection using Proportional-Integral Observer for application to elastic mechanical structures," in IFAC - IMACS Multiconference on Computational Engineering in Systems Applications, CESA'96, Lille, France, 1997.

[10] K. Busawon and P. Kabore, "Disturbance attenuation using proportional integral observers," International Journal of Control, vol. 74, no. 74, pp. 618-627, 2001.

[11] B. Shafai, C. Pi, and S. Nork, "Simultaneous disturbance attenuation and fault detection using proportional integral observers," in American Control Conference, ACC, Anchorage, AK, 2002, pp. 1647-1679.

[12] Y. Xiong and M. Saif, "Unknown disturbance inputs estimation based on a state functional observer design," Automatica, vol. 39, no. 8, pp. 1389-1398, 2003.

[13] D. van Schrick and C. Baspinar, "Some aspects on the proportionalintegral observer in the field of system supervision," in 14th IFAC Word Congress, Beijing, P. R. China, 1999, pp. 539-544.

[14] R. Murray-Smith and T. Johansen, Multiple model approaches to modelling and control, R. Murray-Smith and T. Johansen, Eds. London: Taylor \& Francis, 1997.

[15] D. Filev, "Fuzzy modeling of complex systems," International Journal of Approximate Reasoning, vol. 5, no. 3, pp. 281-290, 1991.

[16] M. Takagi and M. Sugeno, "Fuzzy identification of systems and its application to modelling and control," IEEE Transactions on Systems Man and Cybernetics, vol. 15, no. 1, pp. 116-132, 1985.

[17] K. Tanaka and M. Sugeno, "Stability analysis and desing of fuzzy control systems," Fuzzy Sets and Systems, vol. 45, no. 2, pp. 135156, 1992.

[18] R. Bergstern, P. Palm and D. Driankov, "Observers for Takagi-Sugeno fuzzy systems," IEEE Transactions on Systems, Man and Cybernetics, vol. 32, no. 1, pp. 114-121, 2002.

[19] R. Babuska, Fuzzy modeling for control. Boston: Kluwer Academic Pulishers, 1998.

[20] A. Venkat, P. Vijaysai, and R. Gudi, "Identification of complex nonlinear processes based on fuzzy decomposition of the steady state space," Journal of Process Control, vol. 13, no. 6, pp. 473-488, 2003.

[21] L. Thiaw, K. Madani, R. Malti, and G. Sow, "Implementation of recurrent multi-models for system identification," in 4th International Conference on Informatics in Control, Automation and Robotics, ICINCO, Angers, France, 2007, pp. 314-321.

[22] P. Gawthrop, "Continuous-time local state local model networks," in IEEE Conference on Systems, Man \& Cybernetics, Vancouver, Canada, 1995, pp. 852-857.

[23] E. P. Gatzke and F. J. Doyle III, "Multiple model approach for CSTR control," in 14th IFAC World Congress, Beijing, P. R. China, 1999, pp. 343-348.

[24] G. Gregorcic and G. Lightbody, "Control of highly nonlinear processes using self-tuning control and multiple/local model approaches," in 2000 IEEE International Conference on Intelligent Engineering Systems, INES 2000, Portoroz, Slovenie, 2000, pp. 167-171.

[25] F. Uppal, R. Patton, and M. Witczak, "A hybrid neuro-fuzzy and decoupling approach applied to the DAMADICS benchmark problem," in Symposium on Fault Detection, Supervision and Safety for Technical Processes, SAFEPROCESS'03, Washington, DC. USA, 2003.

[26] R. Orjuela, B. Marx, J. Ragot, and D. Maquin, "State estimation for nonlinear systems using a decoupled multiple model," International Journal of Modelling Identification and Control (to be published), 2008.

[27] R. Orjuela, D. Maquin, and J. Ragot, "Nonlinear system identification using uncoupled state multiple-model approach," in Workshop on Advanced Control and Diagnosis, ACD'2006, Nancy, France, 2006.

[28] S. Boyd, L. El Ghaoui, E. Feron, and V. Balakrishnan, Linear Matrix Inequalities in System and Control Theory, ser. SIAM studies in applied mathematics. Philadelphia, P.A.: SIAM, 1994.

[29] J. Löfberg, "YALMIP : A toolbox for modeling and optimization in MATLAB," in Computer Aided Control Systems Design, CACSD, Taipei, Taiwan, 2004, pp. 284- 289

[30] J. F. Sturm, "Using SeDuMi 1.02, A Matlab toolbox for optimization over symmetric cones," Optimization Methods and Software, vol. 11, no. 1, pp. 625-653, 1999. 